\title{
Entrelacs
}

Cinéma et audiovisuel

\section{Le genre dans The Rocky Horror Picture Show ou la liberté préservée par la perversion}

Filaire Marc-Jean

\section{(2) OpenEdition}

Journals

Édition électronique

URL : http://journals.openedition.org/entrelacs/363

DOI : $10.4000 /$ entrelacs.363

ISSN : 2261-5482

Éditeur

Éditions Téraèdre

Référence électronique

Filaire Marc-Jean, « Le genre dans The Rocky Horror Picture Show ou la liberté préservée par la perversion ». Entrelacs [En ligne], 9 | 2012, mis en ligne le 22 octobre 2012, consulté le 21 avril 2019. URL : http://journals.openedition.org/entrelacs/363; DOI : 10.4000/entrelacs.363

Ce document a été généré automatiquement le 21 avril 2019

Tous droits réservés 


\title{
Le genre dans The Rocky Horror Picture Show ou la liberté préservée par la perversion
}

\author{
Filaire Marc-Jean
}

1 Alors qu'à sa sortie en 1975 le film n'eut presque aucun succès, l'expression de film culte estampille aujourd'hui l'impact culturel du Rocky Horror Picture Show. Le scandale, en revanche, ne l'a pas épargné; le goût manifeste pour la transgression ne pouvait évidemment pas plaire à tout le monde. Il est certain que l'ambiguïté sexuelle de Frank-NFurter, le personnage principal, a dû troubler le public, car encore aucun film rock n'avait tenté une telle remise en cause des frontières de genre. Si l'on s'en tient au constat de scandale, il faut comprendre ce qui motive la résistance du grand public et l'engouement d'un autre public, délibérément attaché au caractère subversif de l'œuvre de Jim Sharman. Dans cette œuvre, où s'enchaînent des événements sans lien apparent, il manque assurément une cohérence narrative, que la présence de Frank-N-Furter dissimule par une présence quasi constante propre à réunifier la diégèse autour de sa seule présence délirante. Ainsi, l'ordre de cette œuvre singulière repose sur le désordre insufflé par un personnage délibérément transgressif, qui porte atteinte aux normes hétérocentrées de la société américaine des années 70. Néanmoins, on pourrait trouver peu subversive une fictionnalisation bien ordonnée même si celle-ci semble faire le portrait d'un personnage transgressif ; l'intérêt du Rocky Horror Picture Show repose en partie sur la capacité à ne pas normaliser sa forme filmique et à conserver une part de folie dans la structuration scénaristique. La transgression de genre propre au personnage principal concentre donc la charge globale d'un film qui fonde toute son esthétique sur la transgression des modèles esthétiques tout autant que moraux. 


\section{Frank, un modèle de perversion?}

2 Tout le film s'organise autour du personnage extravagant de Frank, de son apparition à sa mort, il est le cœur du Rocky Horror Picture Show. Et si le spectateur est touché de sa fin tragique, il n'en demeure pas moins que, tout au long du film, le savant fou apparait animé d'une perversité évidente. Celle-ci s'inscrit dans l'idée doxale d'une jeunesse évidemment dégénérée et irrespectueuse, dont les signes sont la drogue, le rock et le sexe. Si la drogue peu présente est rapidement évoquée, le rock occupe une place privilégiée autant dans l'esthétique visuelle que sonore du film. Cependant, il faut remarquer que le rapport établi avec le rock 'n' roll est ambigu et non simplement citationnel ou référentiel ; le meurtre violent de Frank à l'encontre d'Eddie (Meat Loaf) semble poser une distance avec la culture rock de manière plutôt cynique. Pourquoi faire apparaître cet ancien amant de Frank jaillissant sur sa moto si c'est pour qu'il soit éliminé dans les minutes qui suivent? Le personnage de Frank s'établit par ce meurtre dans une posture dissidente : avec la référence au rock, le scénario se construit en marge de la culture doxale pervertissant le référent que le film convoque et s'en distinguant par l'ironie d'un crime. Le film affirme sa revendication transgressive en faisant appel à la culture rock, symbole d'anticonformisme et du refus de la norme bourgeoise ; ainsi, il se constitue certes comme oeuvre de transgression mais aussi comme film d'hypertransgression, plus transgressif encore que ce que la doxa réprouve déjà. A cela s'ajoute également le thème de la sexualité, laquelle est vécue par Frank sur le mode transgressif également. Avec le couple de Janet et Brad et les scènes d'intrusion nocturne dans leur lit respectif, Frank nie la frontière normative établie entre les sexualités, comme il nie la distinction traditionnelle entre masculinité et féminité, sa sexualité fait voler en éclats les limites de la culture bourgeoise hétérocentrée. Ainsi, le film de Jim Sharman trouve son originalité dans l'affirmation de sa dissidence et ce de manière explicite et revendiquée.

3 La dynamique transgressive du film trouve sa manifestation également dans le déroulement même du scénario et l'approche diachronique de la sexualité au fil du récit. La représentation du désir est établie, au tout début du film, de façon parodique par le cliché humoristique de l'amour marital et de la relation désérotisée du jeune couple plus frustré que candide, qui glousse niaisement au mariage d'amis, en rêvant de prendre leur place. Le point de départ de la narration est aussi le commencement d'un parcours initiatique vers une libération psychologique, dont la scène ingénue de l'église est le contre-exemple, l'anti-modèle à inverser : les deux tourtereaux égarés se trouvent pris dans la cage au fol désir, mais cette prison se fait le lieu de leur renaissance dans un corps plus épanoui, en accord avec leurs pulsions jusque là retenues. L'un et l'autre sont, dans un premier temps, contraints par leurs hôtes étranges d'abandonner les vêtements qui leur donnent une allure si kitsch; dans cette tenue légère, ils trouvent peu à peu leur aise, ne revenant jamais au cours du film à une tenue vestimentaire traditionnelle. L'étape suivante est celle de l'adultère et du multipartenariat pour Janet et de l'homosexualité pour Brad, expériences transgressives par excellence au sein de la société occidentale contemporaine. Puis, l'initiation continue et met en scène la métamorphose complète par le biais de la statufication et le déguisement imposé aux corps figés, lesquels renaissent à la vie pleinement épanouis dans leur nouvelle allure camp. Tout ce parcours s'accompagne d'une mise en mots du désir, qui avait commencé avec la chanson « Sweet 
Transvestite » (Tendre travesti), qui avait continué avec le duo de Janet et Rocky, « Touch Me » (Touche-moi), dont le propos est explicite, et qui finissait avec « Don't Dream It, Be It » (Ne le rêve pas, sois-le) et "Wild et Untamed Thing " (Quelque chose sauvage et indompté). Ainsi, tout le film revendique une volonté d'échapper à une morale contraignante, que Magenta et Riff Raff imposent néanmoins à la fin de l'histoire en tuant Frank et en reproduisant de manière parodique le couple austère de colons américains peint par Grant Wood en 1930. La libération sexuelle revendiquée par Frank, porte-parole de tout le film, achoppe, à la fin, sur la pruderie de la société bien-pensante et sur la culture d'une jouissance frustrée qui fonde la culture chrétienne ${ }^{1}$. Le Rocky Horror Picture Show de Jim Sharman n'aura été qu'un show, une festive parenthèse de liberté, refermée avant un retour à la normalité grise et hétéronormée.

Pourtant, pendant un moment, le souffle libertaire d'une transgression joviale est passé sur le spectateur du film et celui-ci a pris contact avec une vision décalée de la sensualité et ce grâce à l'initiation du très déroutant Frank, qui est un modèle de subversion érotique, difficilement étiquetable : la bisexualité du personnage n'est ni réductible à une revendication politique ni assimilable à une ambivalence équilibrée ; plus simplement, on peut faire le constat qu'au cours de la diégèse Frank manifeste un désir à la fois pour Janet, Brad et Rocky. Cette attitude érotique, qui fait fi des frontières traditionnelles, place le maître des lieux dans une posture psychologique décalée par le fait même de refuser les rappels moralisateurs des deux fiancés ingénus mais aussi curieux de nouveauté. Le personnage bisexuel ou pansexuel se définit comme un objectivateur du désir, il aide à la prise de conscience du fantasme refoulé et à sa réalisation nécessaire à un épanouissement personnel. Il offre à Janet l'opportunité d'une sexualité libérée du cadre moral de la fidélité imposée et non choisie ; à Brad, il donne le moyen d'expérimenter ses désirs homoérotiques. La pansexualité de Frank est l'élément nécessaire à la métamorphose des autres personnages. Mais il ne faudrait pas réduire ce pouvoir d'influence à une forme supérieure d'égotisme, où la jouissance individualiste viendrait saper les fondements des liens sociaux. Au contraire, Frank donne accès à une nouvelle conception du groupe social, dans lequel la hiérarchisation traditionnelle des sexes ne fonctionne plus. Si à l'arrivée au château, Brad se présentait comme le protecteur naturel de sa fiancée, cette figure du mâle est rapidement établie comme artificielle et sans réalité effective : Brad est impuissant devant ses hôtes et surtout devant Frank, qui se moque d'être défini comme un être mâle ou femelle, il est avant tout un être désirant et sans contrainte. Ainsi, hommes et femmes du public psychédélique portent des tenues similaires, ont une gestuelle semblable : les codes de reconnaissance sociaux ne fonctionnent plus dans le château de Frank-N-Furter, on est membre de la communauté et non un être catégorisable par son sexe. Ainsi, se met en place un fonctionnement général trans-sexuel, où tout être passe outre les frontières de son sexe biologique, de son sexe social et de la sexualité qui y est rattachée par la tradition. Sur le modèle de Frank, chaque occupant peut être homme et femme en même temps, porter des bas avec un haut-de-forme et aimer qui bon lui semble. Frank incarne le rêve libertaire des années 70 mais son exécution finale annonce le retour à la moralisation nixonienne que les années sida vont remettre au goût du jour au cours de l'ère réactionnaire du reaganisme. Son pouvoir subversif ne peut advenir que dans un film qui joue avec les codes du cinéma fantastique, comme si la liberté d'un désir épanoui ne pouvait qu'être surnaturelle ou extraterrestre. La joie qu'insuffle le personnage à son environnement porte en elle une part de pessimisme indéniable sur l'évolution des mentalités. 


\section{Le combat contre les modèles}

Si le film de Jim Sharman semble tant s'opposer aux modèles traditionnels, c'est qu'il les pense dangereux pour l'individu et la liberté de pensée, d'expression et de jouissance. Dès lors, il s'agit de faire voler en éclats tous les types de modèles qui seraient susceptibles de se manifester, afin d'établir une œuvre filmique intrinsèquement en rupture avec tout référent et ce au prix d'un baroquisme revendiqué. La référence artistique qui convoque le plus clairement la morale traditionaliste est évidemment le tableau de Grant Wood, American Gothic (1930), dont une copie apparaît derrière Riff Raff à l'arrivée de Janet et Brad au château, mais aussi une mise en scène avec le couple de paysans devant la porte de l'église au tout début du film. Enfin, la transposition pseudo-futuriste de la même image avec Riff Raff et Magenta révélant leur vrai visage à la fin du film montre l'exubérante relecture de ce tableau qui a servi de modèle et aux Américains qui revendiquant l'héritage des anciens colons et à leur opposants. Le choix de cette œuvre est d'autant plus approprié pour un jeu parodique que Grant Wood lui-même a laissé se multiplier les commentaires les plus contradictoires sur un tableau qu'il a voulu ironique : un paysan en veston et une femme portant camée et robe élégante ne sont pas les modèles les plus justes pour représenter un couple de paysans, sachant en outre que les modèles du peintre sont sa sœur et son dentiste. Dans The Rocky Horror Picture Show, cette référence ouvre et ferme le film rappelant que tout ce qui existe hors du film, avant lui et après lui, est associable à ce tableau, associé désormais à une idéologie qui impose sa norme morale comme indiscutable, sur le mode dogmatique. En convoquant de manière répétée l'œuvre de Wood, Jim Sharman réactive la portée ironique du projet initial de l'artiste. D'autres images sont associées à la morale oppressive au fil du récit et chacune est ridiculisée. Riff Raff et Magenta eux-mêmes, lors de leur ultime apparition sont des extraterrestres aux costumes et aux armes comiques : le pistolet en forme de fourche en est l'exemple le plus visible. Parmi la foule des adeptes de Frank, il faut aussi être attentif à la variété des âges et constater que l'association convenue de la vieillesse et de la sagesse ne fonctionne évidemment pas. Les présents participent tous de la folie communicative de Frank, ils dansent le Time Warp sans hiérarchisation des âges ni des sexes : les gestes sont les mêmes pour tous. Le Dr Scott lui-même, respectable professeur dont l'honorabilité semble renforcée par le siège roulant qui lui permet de se déplacer, laisse finalement les pulsions se libérer sous l'influence de Frank. Ainsi, les supports traditionnels de la respectabilité sont raillés et pris dans le mouvement de l'extravagance libératrice. Le criminologue lui-même, dans sa posture externe de narrateur, subit l'influence transgressive du château et se retrouve à danser sur son bureau. Le spectateur également souhaite peut-être se laisser entraîner par le rythme. Cependant, la charge moralisatrice qui s'abat sur Frank et l'anéantit paraît miner tous les efforts provocateurs de celui-ci et faire de la violence de l'oppression la puissance la plus forte, celle à laquelle il n'est possible de résister que temporairement, avant d'être rattrapé. La graine que le démiurge fou a semé dans l'esprit du spectateur a-t-elle des chances de croître?

Il est certain que les adeptes de séries B constituent le premier public avec lequel s'établit une connivence intellectuelle par tout un jeu de références et de pastiches. Ceux qui savent reconnaitre les momies des mauvais films d'horreur dans la naissance de Rocky, l'écho à Docteur $\mathrm{X}$ ou à Flash Gordon, le nom de Janette Scott dans ceux de Janet et de $\mathrm{D}^{\mathrm{r}}$ Scott, ceux-là sont déjà des convertis à la folie des Transylvaniens et il n'est rien besoin 
de leur prouver : ils constituent un parterre de convaincus et sont assimilables à ceux qui participent au Time Warp ou au public du cabaret applaudissant aux adieux de Frank. Pour les autres, peut-être faudra-t-il se contenter des références plus célèbres : les échos littéraires jouent également comme points de repères, la créature de Frankenstein et Dracula sont comme les ancêtres sérieux de Rocky et de Frank. Mais à quoi servent ces références? Faut-il voir dans leur convocation le ralliement à un certain classicisme de fond à laquelle le Show n'échapperait pas? Ainsi, le carcan idéologique se retrouverait à la source même de la transgression et donc la réduirait à un simple jeu érudit. On ne peut se résoudre à penser que le film culte a pu connaître une telle postérité sans se faire le médiateur d'une véritable révolte culturelle. Rien qu'en revenant aux références littéraires, il s'établit une dynamique délibérément libertaire : la créature de Mary Shelley n'est-elle pas l'œuvre du "Prométhée moderne ", comme l'affirme le sous-titre du roman? Dracula n'est-il pas l'outrepasseur des barrières victoriennes? L'un et l'autre sont des voyageurs qui se rient des frontières géographiques ou morales et sont donc les ancêtres symboliques de Frank; le motif de la cape assure une filiation symbolique, tout autant que les intrusions nocturnes dans les chambres. La convocation ludique des références participe donc pleinement de l'élan transgressif du Rocky Horror Picture Show. Les modèles culturels, aussi ancrés qu'ils soient dans l'histoire collective, sont réactivés à des fins militantes pour combattre les scléroses de la tradition, lorsque celle-ci tombe dans l'autosatisfaction et le figement idéologique.

7 Pour réaliser pleinement cet objectif de révolte identitaire, le film empreinte également la voie toujours décriée du discours libertaire de la sexualité. Ici, s'ajoute à la revendication d'une polysexualité la mise à mal du modèle hétérosexuel, pris dans l'étau des images du premier et du dernier couples montrés à l'écran : à un bout du film on rit de l'épaisse candeur de Janet et Brad, à l'autre extrémité Riff Raff et Magenta forment un couple effrayant à la morale sans concession. Entre ces deux images une autre se développe, en rupture avec les schémas traditionnels; en effet, la séparation hiérarchisante des sexes n'a plus lieu d'être dans le château de Frank, les tenues sont similaires sans distinction claire de genre ou potentiellement érotiques mais non au détriment des femmes, si Columbia a une tenue de soubrette coquine, Rocky est un pur objet de désir, créé pour sa perfection plastique par Frank. Tout le monde est donc un possible objet de désir au regard des autres, libérant par là même le regard de chacun et les pulsions de tous. L'ajout principal du film par rapport au spectacle originel, tout ce qui concerne Eddie, va dans le même sens : si le rock est symbole de transgression dans les années 70 - et même de transgression sexuelle avec le glam-rock - le rock 'n' roll ne l'est guère sur le plan érotique et Eddie (Meat Loaf) représente une image de la misogynie que Frank élimine sans remords. Son crime est un acte de revendication contre le rocker, figure encore trop marquée par l'idéologie hétéronormée et éliminée de la manière la plus outrancière et immorale, par un meurtre sanglant et gratuit: n'est-ce pas là une manière transgressive de rompre avec la morale, en caricaturant son fonctionnement même ? Le film affirme donc son objectif révolutionnaire en tentant d'aller toujours plus loin dans son action iconoclaste et en outrepassant même ce qui semblait déjà être pervers. Ainsi, se constitue une morale libertaire de l'outrance, fondée sur une esthétique de la perversion des modèles. 


\section{Une esthétique de la perversion pervertie}

8 L'objet filmique qu'est le Rocky Horror Picture Show affirme sa rupture totale et se construit comme une œuvre hors normes. L'impression d'incohérence frappe le spectateur la première fois, le scénario paraît constitué de bric et de broc: le mariage n'a guère de lien avec l'histoire au château de Frank-N-Fuster, pas plus que le Time Warp avec la création de Rocky, quant à l'arrivée du Dr Scott ou à la trahison finale de Riff Raff et Magenta, elles sont reliées à la nuit sulfureuse de Janet et Brad de manière aussi artificielle que l'envol final du château avec le dîner cannibale : seule la continuité des images, la présence des mêmes personnages et la volonté du spectateur à trouver une unité assure à la diégèse de ne pas se disloquer en sketchs indépendants. Pourtant, malgré ce désordre apparent, le film est une œuvre culte faisant fi de l'évidente cacophonie scénaristique. Remarquons, néanmoins, qu'il est donné au criminologue la posture du narrateur, posture par nature ordonnatrice d'un récit, dont il garantit l'unité. Dans le film, on peut se demander si ce personnage remplit bien l'attente que l'on a à l'égard de sa position; dans la mesure où il se fait le commentateur des événements, il se place en deçà des événements rapportés et non au-dessus, et s'il ne semble pas les découvrir, il n'en paraît pas non plus le maître. Ainsi, dans la composition même de l'acte narratif du film, le schéma choisi est subverti et transforme l'instance démiurgique en un témoin extérieur mais informé comme l'est tout adepte du film culte : comme le spectateur averti, il connaît l'issue tragique de l'histoire mais se délecte à la revivre de manière très intense, en s'y impliquant même physiquement : son Time Warp debout sur le bureau est l'incarnation dans le film des fans costumés à la manière des personnages lors des rediffusions. Dès lors, on est en droit de se demander s'il était possible que le film soit ordonné selon une structuration narrative traditionnelle. Tout ordre visible ne risquait-il pas de porter atteinte à l'objectif transgressif d'« un des seuls films conçus majoritairement par des gays et dans une optique gay ", " plaidoyer bisexuel, voire transsexuel ${ }^{2}$ » ? Le désordre formel participe de la revendication subversive et donne à voir un objet délirant non identifié « où l'outrance domine de bout en bout ${ }^{3}$ ». Le désordre n'est pas un état de fait dans une œuvre mal achevée mais un désordre affirmé propre à solliciter une attitude active du spectateur.

9 Au cœur de l'histoire, on trouve de nombreux indices d'une volonté de révolte, le film s'affirme comme fondamentalement en marge, puisqu'il ne cesse de marteler le motif de l'ailleurs : le château de Frank-N-Furter n'appartient pas à la réalité, il ouvre un espacetemps parenthétique où tous les possibles du fantasme et du fantastique peuvent advenir. Le fonctionnement de la temporalité reprend le principe des films d'horreur, dans lesquels des jeunes gens basculent pendant un temps donné dans un monde d'épouvante où l'effroyable advient au détriment de toutes les règles de la rationalité. Quant au lieu, il est lui aussi aberrant: le château de la peur est un vaisseau spatial venu de la planète Transsexuel dans la galaxie de Transylvanie et se trouve au cœur de la forêt effrayante des contes de fée ou des films d'horreur. Forêt et portes du château sont des barrières qui répètent que le voyage entrepris par le «couple ingénu et propret formé par Janet et Brad, concentré de niaiserie issu de l'Amérique nixonienne ${ }^{4}$ ", mais aussi par le public, est un voyage pour un ailleurs fantastique. Pourtant, le château de la subversion est luimême victime de son désir subversif, comme s'il ne pouvait qu'engendrer sa propre subversion, il fait apparaître en son sein les traîtres à sa propre cause. Ce retournement de situation annonce peut-être un échec sur le plan de la morale mais garantit l'ambition 
subversive de l'œuvre : toute subversion est potentiellement à subvertir, sinon elle devient norme.

Pour en revenir, finalement, au personnage même de Frank, il convient de reconnaître que le protagoniste marqué du sceau de la perversion affirme sa propre nécessité intellectuelle : le pervers dit plus que sa perversion, il en appelle à une remise en cause de la morale, à une refonte de son champ d'action. Certes, le personnage transgressif redit la nécessité de la morale, de son rôle correctif, mais il sollicite la rupture, le refus d'acceptation naïf. "Défi vivant à la pudibonderie comme aux règles culturelles et sociales $^{5}$ ", Frank-N-Furter, en un ultime spectacle d'adieux, accepte sa disparition nécessaire et ce avec une grâce tout aussi camp que tragique: son dernier spectacle devant un public enthousiaste de fantômes vieillissant constitue le point crucial de la redéfinition du pervers à la cruauté narcissique en un pervers positif. Sa métamorphose, à l'heure où son maquillage se défait, révèle qu'il a moins contaminé qu'il n'a libéré. En se constituant en modèle des contre-modèles, il offre aux spectateurs un exemple de liberté affirmée, excessive certes mais néanmoins nécessaire face aux forces revenantes de l'oppression moralisatrice. Doit-on craindre que la mort du personnage soit l'affirmation d'un pessimisme idéologique? C'est possible. Toutefois, une lecture moins sombre est encore possible. Dans les dernières images du film, Brad, Janet et le $\mathrm{D}^{\mathrm{r}}$ Scott sont des " insectes", selon le criminologue, qui « rampent à la surface de la Terre", des êtres qu' " on appelle la race humaine, perdus dans le temps, l'espace et la signification », mais ils portent encore le maquillage, les guêpières et les bas que leur a donné Frank. Ainsi, ils sont exclus du château de la subversion mais ils en gardent les marques, tout n'est donc pas perdu s'il reste ne serait-ce que les costumes qui refusent les catégories sociales et sexuelles. D'ailleurs, c'est en costumes que les adeptes du Rocky Horror Picture Show font perdurer l'esprit transgressif du film, lorsqu'ils s'adonnent à ces séances extravagantes où projection d'eau et lancer de riz transforme la diffusion en carnaval, et l'on sait que pendant carnaval il n'est plus de normes qui tiennent.

11 Ainsi, c'est sur les costumes extravagants et fortement érotisés que s'achève le film, affirmant par ce moyen l'indistinction à laquelle en sont arrivés " ces âmes benoîtes et gourmées ${ }^{6} »$ de Janet et Brad. La perversion des normes s'est accomplie et le mode de représentation traditionnelle des sexes ne fonctionnent plus. Féminin et masculin ne sont plus des catégorisants pertinents, car le désordre a contaminé la réalité, pervertissant de manière joyeuse et positive l'ordre normatif. La réussite de ce film est sûrement d'avoir réussi à trouver des adeptes hors du seul public gay: "les fans de fantastique et de science-fiction qui le vénèrent pour ses multiples références à leurs genres favoris, font assez peu de cas - lorsqu'ils ne l'ignorent pas totalement - du plaidoyer bisexuel, voire transsexuel, que le film développe avec une ferveur tonitruante ${ }^{7} »$. Pour proposer une redéfinition du genre, hors de la seule tension binaire entre masculin et féminin, il convient de revoir de fond en comble l'imagerie populaire sur le sexe, The Rocky Horror Picture Show ose le grand ménage, la perversion affichée et revendicative, mais cette dernière ne se pose pas en nouvelle force d'oppression, elle dit ses pouvoirs, ses faiblesses et le risque d'être elle-même pervertie, ce qui est le seul moyen de rester prévoyant face aux violences d'une norme qui se veut univoque et intellectuellement mortifère. 


\section{NOTES}

1. Sur cette question, on consultera avec profit Le Souci des plaisirs de Michel Onfray, Flammarion, J'ai lu, 2008.

2. Voir l'article de BBJane, "Le diptyque culte de Jim Sharman "publié sur le site <http:// fearsforqueers.blogspot.com/>.

3. Ibid.

4. Ibid.

5. Ibid.

6. Ibid.

7. Ibid.

\section{RÉSUMÉS}

Le genre dans The Rocky Horror Picture Show ou la liberté préservée par la perversion

Frank-N-Furter, le délirant protagoniste du Rocky Horror Picture Show, bouscule par ses jeux érotiques les normes hétéronormées de la société bourgeoise. Son goût apparent pour la violation des règles doxales en fait un parangon de perversion, non pour répandre le vice mais pour ébranler la bien-pensance et inciter à discuter ses limites sans les considérer comme acquises. Ainsi, la transgression se fait la garante d'une perpétuelle remise en cause idéologique, au risque d'engendrer l'ennemi qui peut l'anéantir. En refusant d'appartenir à une catégorie définie de genre, Frank-N-Furter pose sa résistance comme un acte militant qui, par-delà l'identité sexuelle, se veut délibérément libertaire.

\section{AUTEUR}

\section{FILAIRE MARC-JEAN}

À l'université de Nîmes, Marc-Jean Filaire enseigne la littérature comparée et les rapports entre texte \& cinéma. Il est l'auteur de L'Ado, la folle et le pervers. Images et subversion gay au cinéma (H\&O éditions, 2008) et de divers articles consacrés à des films, notamment en lien avec les thèmes d'homosexualité et de vampirisme. Il tient la rubrique « Derrière les masques : Homollywood » sur le site Les Toiles Roses. En juin 2010, il a organisé le colloque international « Marguerite Yourcenar \& la culture du masculin » à l'université de Nîmes (Lucie éditions, octobre 2011). 